\title{
Injury promotes sarcoma development in a genetically and temporally restricted manner
}

\author{
David Van Mater, ${ }^{1}$ Eric Xu, ${ }^{2}$ Anupama Reddy, ${ }^{3}$ Leonor Añó, ${ }^{4}$ Mohit Sachdeva, ${ }^{2}$ Wesley Huang, ${ }^{2}$ \\ Nerissa Williams, ${ }^{2}$ Yan Ma, ${ }^{2}$ Cassandra Love, ${ }^{3}$ Lanie Happ, ${ }^{3}$ Sandeep Dave, ${ }^{3}$ and David G. Kirsch ${ }^{2,4}$ \\ 'Department of Pediatrics, Division of Hematology-Oncology, ${ }^{2}$ Department of Radiation Oncology, ${ }^{3}$ Department of \\ Medicine, Division of Hematologic Malignancies and Cellular Therapy, and ${ }^{4}$ Department of Pharmacology and Cancer \\ Biology, Duke University Medical Center, Durham, North Carolina, USA.
}

\begin{abstract}
Cancer results from the accumulation of genetic mutations in a susceptible cell of origin. We and others have also shown that injury promotes sarcoma development, but how injury cooperates with genetic mutations at the earliest stages of tumor formation is not known. Here, we utilized dual recombinase technology to dissect the complex interplay of the timing of Kras ${ }^{\mathrm{G} 12 \mathrm{D}}$ activation, p53 deletion, and muscle injury in sarcomagenesis using a primary mouse model of soft tissue sarcoma. When mutations in oncogenic Kras and p53 are separated by 3 weeks, few sarcomas develop without injury. However, the transformation potential of these tumor-initiating cells can be unmasked by muscle injury. In the absence of Kras mutations, injury of the muscle with global deletion of $\mathrm{p} 53$ results in sarcomas with amplification of chromosomal regions encompassing the Met or Yap1 gene. These findings demonstrate a complex interplay between the timing of genetic mutations and perturbations in the tumor microenvironment, which provides insight into the earliest stages of sarcoma development.
\end{abstract}

Conflict of interest: David Kirsch is on the scientific advisory board of Lumicell Inc., which is a company commercializing intraoperative imaging, and is a cofounder of XRAD Therapeutics, which is developing radiosensitizers.

Submitted: July 19, 2018

Accepted: August 30, 2018

Published: October 18, 2018

Reference information: JCI Insight. 2018;3(20):e123687. https://doi.org/10.1172/jici. insight.123687.

\section{Introduction}

Soft tissue sarcomas arise from a mesenchymal cell of origin and represent more than 70 different tumor types. Soft tissue sarcomas can be broadly classified as those harboring single genetic alterations such as a translocation (i.e., simple karyotype) and those with a constellation of poorly defined genetic changes (i.e., complex karyotype) (1). Among soft tissue sarcomas with a complex karyotype, we have previously described a mouse model of undifferentiated pleomorphic sarcoma (UPS) arising from the muscle after simultaneous activation of $\operatorname{Kras}^{\mathrm{G} 12 \mathrm{D}}$ and loss of p53 $(2,3)$.

Recently, Young et al. utilized dual recombinase technology to explore the impact of sequentially mutating Kras and p53 in a mouse model of sarcoma (4). Dual recombinase technology utilizes 2 different site-specific recombinases (Cre and Flp) to recombine distinct genes in a temporally and spatially restricted manner $(5,6)$. In the experiments conducted by Young et al., the yeast-based Flp recombinase was used to excise a STOP cassette flanked by FRT sites upstream of oncogenic Kras (Kras ${ }^{\text {FSF-G12D }}$ ) via intramuscular (IM) injection of adenovirus expressing a mammalian codon-optimized version of Flp (adeno-FlpO) (7). Temporal control of p53 deletion was regulated by intraperitoneal (IP) tamoxifen administration to activate CreER in cells throughout the mice (Rosa26 $6^{\mathrm{CrER} / \mathrm{CreER}}$ ), thereby causing recombination of LoxP sites flanking exons $2-10$ of $\mathrm{p} 53\left(\operatorname{Trp} 53^{\mathrm{f} / \mathrm{fl}}\right)$. A critical observation from those experiments was that the penetrance and kinetics of sarcoma formation were significantly increased when Kras was activated at the same time as p53 loss as compared with when the timing of Kras activation and p53 loss was uncoupled. The authors concluded that cells harboring activated Kras in the setting of WT p53 have reduced transformation potential, which prevents sarcomagenesis when p53 is subsequently deleted.

We recently used Pax $7^{\mathrm{CreER} /+} ; \operatorname{Trp} 53^{\mathrm{fl} / \mathrm{fl}} ; \mathrm{Kras}^{\mathrm{LSL}-\mathrm{G} 12 \mathrm{D} /+}$ mice to show that following systemic IP tamoxifen administration, most muscle satellite cells remain quiescent after activation of Kras and deletion of p53 (8). However, muscle injury in combination with IP tamoxifen was sufficient to promote sarcoma development at the site of injury (8). Therefore, we generated Rosa26 $\mathrm{CreER/CreER} ; \operatorname{Trp} 53^{\mathrm{fl} / \mathrm{fl}} ; \mathrm{Kras}^{\mathrm{FSF}-\mathrm{G} 12 \mathrm{D} /+}$ (R26 ${ }^{\mathrm{CreER}} ; \mathrm{p}^{\mathrm{H}} 3^{\mathrm{H}}$; $\left.\mathrm{K}^{\mathrm{FRT}}\right)$ mice to investigate whether muscle injury might alter the fate of cells with sequential mutations of 
Kras and p53. Specifically, we wished to determine whether quiescent muscle cells harboring activated Kras in the setting of WT p53 would become activated 3 weeks later by deletion of p53 and IM injection of a potent inducer of muscle injury such as cardiotoxin. We report that cardiotoxin dramatically alters the fate of Kras-mutated cells and almost fully restores their transformation potential. Similar results were obtained if the order of gene mutations was reversed. If p53 was deleted and Kras activation occurred 3 weeks later, then there was a delay in the kinetics and decreased penetrance of sarcoma formation. However, tumors formed with high frequency when muscle injury and Kras activation occurred concurrently 3 weeks after p53 deletion. In control experiments, we observed that Kras mutation is dispensable for sarcoma formation when p53 is globally deleted at the same time as an acute muscle injury with cardiotoxin. Remarkably, no sarcomas formed when the injury was delayed until 21 days after p53 loss. We also performed exome sequencing on Kras- and injury-induced (Kras WT) sarcomas. Kras-induced sarcomas were found to have a very low mutational load, with no recurrent mutations. Injury-induced sarcomas also had low mutational load, with no recurrent mutations in Ras pathway genes, but evaluation of copy number variants (CNVs) revealed that these sarcomas harbored recurrent amplification of regions of chromosomes 6 and 9, which include the Met and Yap1 genes, respectively.

By employing dual recombinase technology to vary the timing of Kras and p53 deletion with respect to muscle injury, our data suggest that the mechanism of injury-induced sarcoma formation is dependent on the timing of gene mutations and injury as well as the genetic context. In the setting of Kras activation, injury acts as a classic promoter as previously reported (8). However, in the setting of WT Kras and global p53 deletion, muscle injury selects for the development of sarcomas with specific chromosomal amplifications that bypass the requirement for Ras mutation. These experiments shed light on the early steps of sarcoma development by revealing the complex interplay between the timing of cooperating gene mutations in transformation and a tumor-promoting stimulus in the microenvironment.

\section{Results}

We initially repeated the elegant sequential mutagenesis experiments described by Young et al. (4). We generated R26 ${ }^{\mathrm{CreER}}$; $\mathrm{p} 53^{\mathrm{fl}}$; $\mathrm{K}^{\mathrm{FRT}}$ mice, which allow for systemic deletion of $\mathrm{p} 53$ following IP tamoxifen injection and localized activation of Kras in response to IM adeno-FlpO injection. As described previously, R26 CreER;

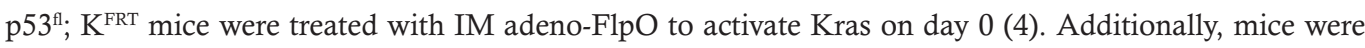
treated with IP tamoxifen on day 0,10 , or 21 to delete p53 in cells throughout the animal at either the same time or after Kras activation (Figure 1A). As in the previous study, we found that sarcoma formation was significantly less robust as the time between Kras activation and p53 deletion was extended from 0 to 21 days (Figure 1, B and C). A sarcoma formed at the IM injection site in only 9\% (1 of 11) of mice when Kras activation and p53 deletion were separated by 21 days. All mice were followed for a minimum of 100 days after tamoxifen administration.

We recently reported that muscle injury accelerates tumor formation in a mouse model of soft tissue sarcoma (8). R26 $6^{\mathrm{CreER}}$; p53 $3^{\mathrm{fl}}$. $\mathrm{K}^{\mathrm{FRT}}$ mice require an IM injection of adeno-FlpO to cause recombination of Kras on day 0, so we considered the possibility that the IM injection causes mild muscle injury that promotes sarcoma formation when administered concurrently with p53 deletion. Because a classic promoter would act only in the context of both initiating genetic mutations (i.e., activation of Kras and loss of p53), injury from the adeno-FlpO injection could explain why the rate of sarcoma formation is considerably lower when p53 is deleted 21 days after Kras activation; any impact from injury would have resolved by the time of p53 deletion. To test this hypothesis, we treated R26 $6^{\mathrm{CrER}} ; \mathrm{p} 53^{\mathrm{fl}} ; \mathrm{K}^{\mathrm{FRT}}$ mice as we had previously with IM adenoFlpO on day 0 and IP tamoxifen on day 21. Additional groups of mice were injected on day 21 with either IM adenovirus expressing GFP (adeno-GFP) or cardiotoxin, a component of cobra venom that induces myonecrosis (positive control for muscle injury). IM injection of adeno-GFP on day 21 did not alter the penetrance or kinetics of soft tissue sarcoma formation (Figure 1, B and C). However, treatment with IM cardiotoxin almost completely restored sarcoma formation at the site of injection (Figure 1, B and C). We assessed muscle histology 7 days following IM injection of adeno-FlpO to determine the extent of damage (Figure 1D). Treatment with adeno-FlpO did not cause appreciable damage to the muscle fibers as compared with cardiotoxin (Figure 1D). We conclude that muscle injury with cardiotoxin is sufficient to restore the transformation potential of Kras-mutated cells when subsequent p53 loss is combined with muscle injury.

We next conducted control experiments to evaluate the requirement for Kras activation in sarcoma formation in $\mathrm{R} 26^{\mathrm{CreER}} ; \mathrm{p} 53^{\mathrm{fl}} ; \mathrm{K}^{\mathrm{FRT}}$ mice. Notably, it has been reported that chronic injury with cardiotoxin is sufficient 
A

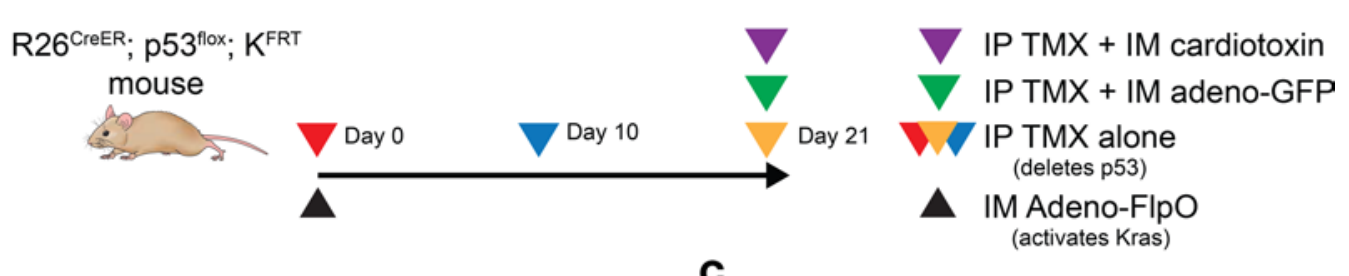

B

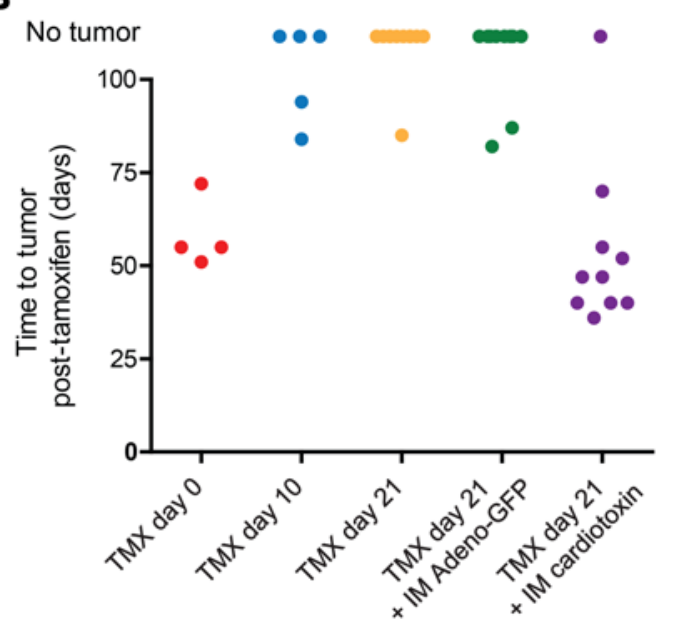

Treatment
C

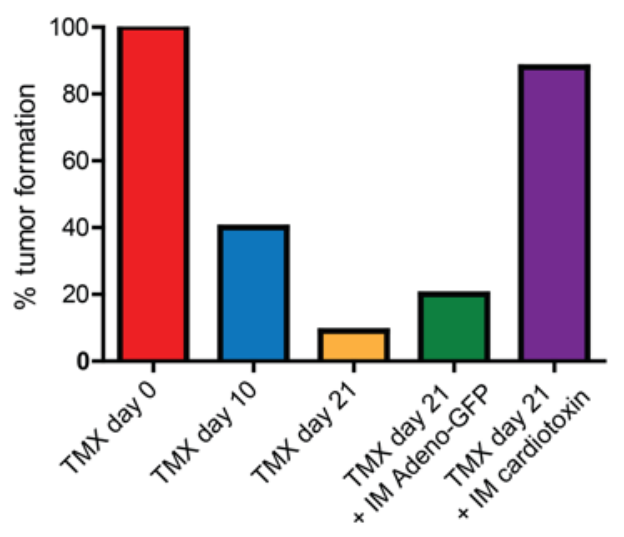

Treatment

D

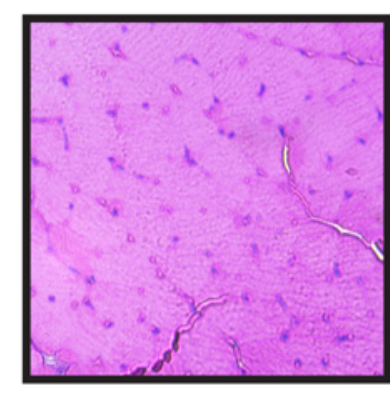

Untreated

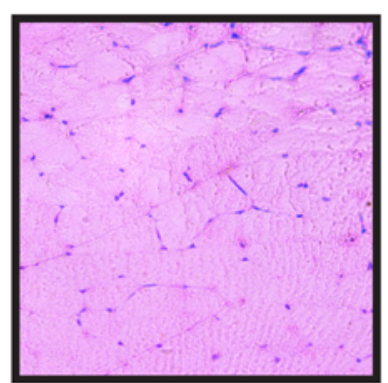

Adeno-FlpO

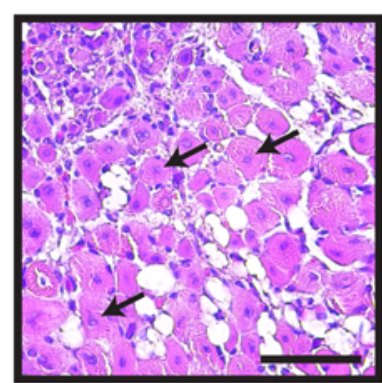

Cardiotoxin

Figure 1. Muscle injury restores the transformation potential of cells harboring activated Kras. (A) Schematic of

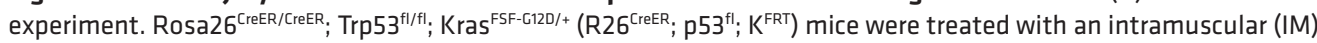
injection of adeno-FlpO to activate Kras on day 0 , followed by treatment with intraperitoneal (IP) tamoxifen to delete p53 on day 0,10 , or 21 . Additional groups of mice were treated with IM adeno-GFP or IM cardiotoxin on day 21 in addition to IP tamoxifen. (B) There is a marked delay in sarcoma formation in R26 ${ }^{\mathrm{CreER}}$; $\mathrm{p} 53^{\mathrm{fl}}$; $\mathrm{K}^{\mathrm{FRT}}$ mice as the time between Kras activation and $\mathrm{p} 53$ deletion is extended from 0 to 21 days. Muscle injury with IM cardiotoxin, but not IM adenoFlpO, restores the transformation potential of Kras-activated, p53-deleted cells. (C) Bar graph presenting the same data as in B. (D) H\&E-stained muscle sections obtained from mice 7 days after the start of the experiment either without treatment or with IM adeno-FIpO or IM cardiotoxin treatment. Evidence of muscle injury and repair (central nuclei noted by arrows) was only observed after cardiotoxin treatment. Scale bar: $50 \mu \mathrm{m}$. TMX, tamoxifen.

to promote sarcoma formation in p53-deficient mice (no activation of Kras) (9). R26 $6^{\mathrm{CreER}} ; \mathrm{p} 53^{\mathrm{fl}}$; $\mathrm{K}^{\mathrm{FRT}}$ mice were treated with IP tamoxifen alone (to delete p53 throughout the animal) and treated with IM cardiotoxin in the hindlimb to mimic acute muscle injury (Figure $2 \mathrm{~A}$ ). Interestingly, $100 \%$ of mice developed sarcomas at the IM injection site despite no Kras activation (Figure 2B). The median time to sarcoma formation was 47 days after cardiotoxin in mice harboring activated Kras and 76 days in mice with WT Kras $(P=0.12$ by log-rank test). Recombination of the Kras ${ }^{\mathrm{FSF}-\mathrm{G} 12 \mathrm{D}}$ allele in the adeno-FlpO-treated mice was confirmed by PCR (Figure 2C). The more rapid sarcoma formation noted in mice with activated Kras was replicated in an independent experiment. R26 CreER; p53 $3^{\mathrm{fl}}$. $\mathrm{K}^{\mathrm{FRT}}$ mice and R26 ${ }^{\mathrm{CreER}} ; \mathrm{p} 53^{\mathrm{fl}}$ littermates were treated identically with IM adeno-FlpO on day 0 and IP tamoxifen/IM cardiotoxin on day 21 (Supplemental Figure 1, A and B; supplemental material available online with this article; https://doi.org/10.1172/jci.insight.123687DS1). Accelerated sarcoma formation was again noted in tumors harboring an activated Kras allele (R26 ${ }^{\mathrm{CreER}} ; \mathrm{p} 53^{\mathrm{fl}} ; \mathrm{K}^{\mathrm{FRT}}$ mice), though this was 
A

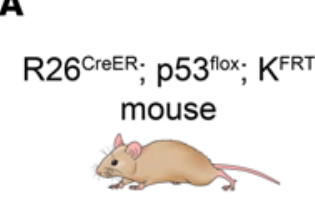

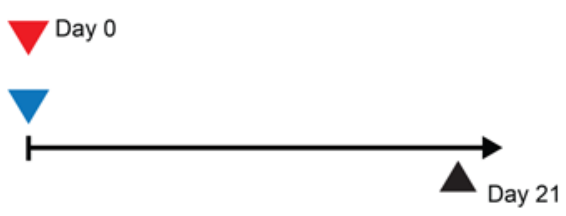

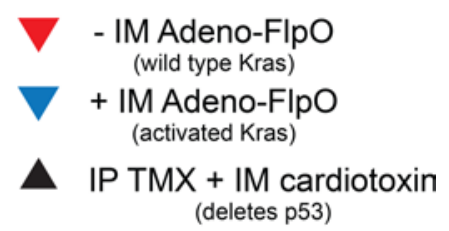

B

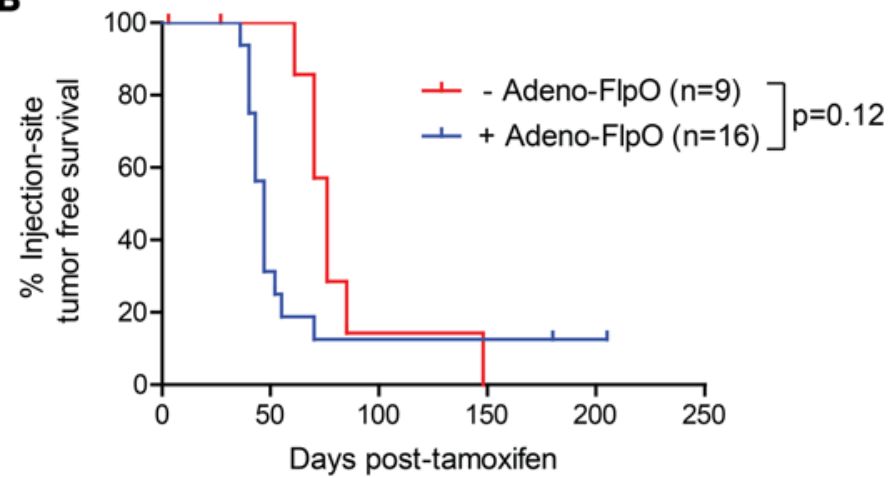

C

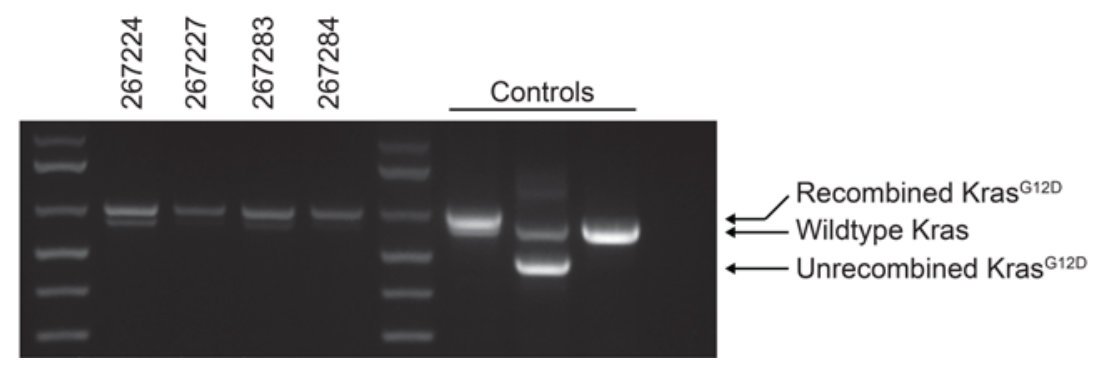

Figure 2. Kras activation is not required for sarcoma formation in the setting of concurrent $\mathrm{p} 53$ loss and injury in R26 ${ }^{\text {CreER }}$; p53 ${ }^{\text {fl; }} \mathbf{K}^{\text {FRT }}$ mice. (A) Schematic of experiment. R2 $6^{\text {CreeR }} ;$ p53 $^{\mathrm{fl}} ; \mathrm{K}^{\mathrm{FRT}}$ mice were either treated or not treated with IM adeno-FIpO to activate Kras on day 0, followed by treatment with IP tamoxifen to systemically delete p53 and IM cardiotoxin on day 21. (B) Survival curve for both treatment groups shows trend toward more rapid sarcoma formation in mice treated with adeno-FlpO and activation of Kras. (C) Cells lines were generated from 4 sarcomas that arose in mice following treatment with IM adeno-FIpO on day 0, and IP tamoxifen and IM cardiotoxin on day 21. PCR-based genotyping reveals recombination of the FRT-STOP-FRT cassette upstream of Kras ${ }^{\mathrm{C12D}}$. Controls show the expected band sizes for recombined $\mathrm{Kras}^{\mathrm{G} 12 \mathrm{D}}$, WT Kras, and unrecombined $\mathrm{Kras}^{\mathrm{G} 12 \mathrm{D}}$.

again not statistically significant (Supplemental Figure 1B). The median time to tumor was 43.5 days in R26 CreER; p53 $3^{\mathrm{fl}}$; $\mathrm{K}^{\mathrm{FRT}}$ mice and 63 days in $\mathrm{R} 26^{\mathrm{CreER}} ; \mathrm{p} 53^{\mathrm{fl}}$ mice $(P=0.36$ by log-rank test). We conclude that Kras activation is not required for sarcoma formation in the setting of systemic p53 loss and concurrent injury. However, sarcomas tend to form more quickly when the Kras allele has already been recombined and activated prior to p53 loss and muscle injury. Of note, a reciprocal experiment was conducted in $\mathrm{R} 26^{\mathrm{CreER}}$; $\mathrm{p} 53^{\mathrm{fl}}$; $\mathrm{K}^{\mathrm{FRT}}$ mice to determine whether Kras activation and injury were sufficient for sarcoma formation. No sarcomas formed in the setting of WT p53, confirming an essential role for p53 loss in this model of sarcoma development (Figure 3, A and B).

We next explored the consequence of p53 deletion prior to Kras activation. We hypothesized that p53 deletion prior to Kras activation would be well tolerated and tumor penetrance would be $\sim 100 \%$ following injection with IM adeno-FlpO to activate Kras. However, there was a marked reduction in tumor penetrance and an increase in time to tumor when p53 deletion preceded Kras activation by 21 days (Figure 4, $\mathrm{A}$ and $\mathrm{B})$. Injury with cardiotoxin fully restored tumor penetrance when coadministered with adeno-FlpO 21 days following p53 deletion, and the time to tumor also decreased such that there was not a statistical difference compared with concurrent p53 deletion and Kras activation (Figure 4B). One possible explanation for this finding is that injury with cardiotoxin eliminates the requirement for Kras activation. This was tested by treating R26 $6^{\mathrm{CreER}} ; \mathrm{p} 53^{\mathrm{fl}} ; \mathrm{K}^{\mathrm{FRT}}$ and $\mathrm{R} 26^{\mathrm{CreER}}$; $53^{\mathrm{fl}}$ littermates with tamoxifen on day 0 , followed 
A

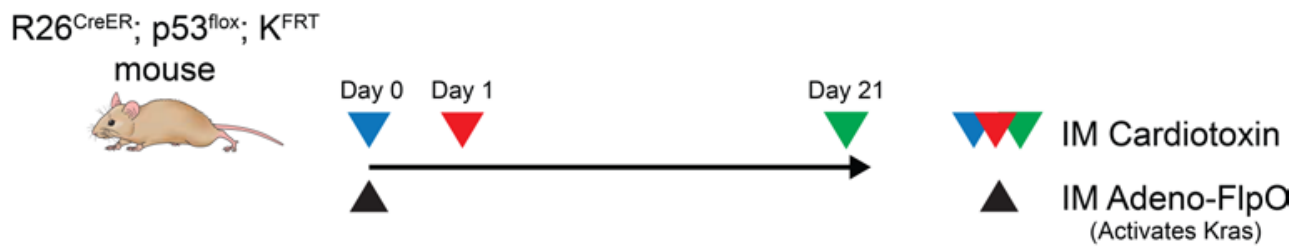

$\mathbf{B}$

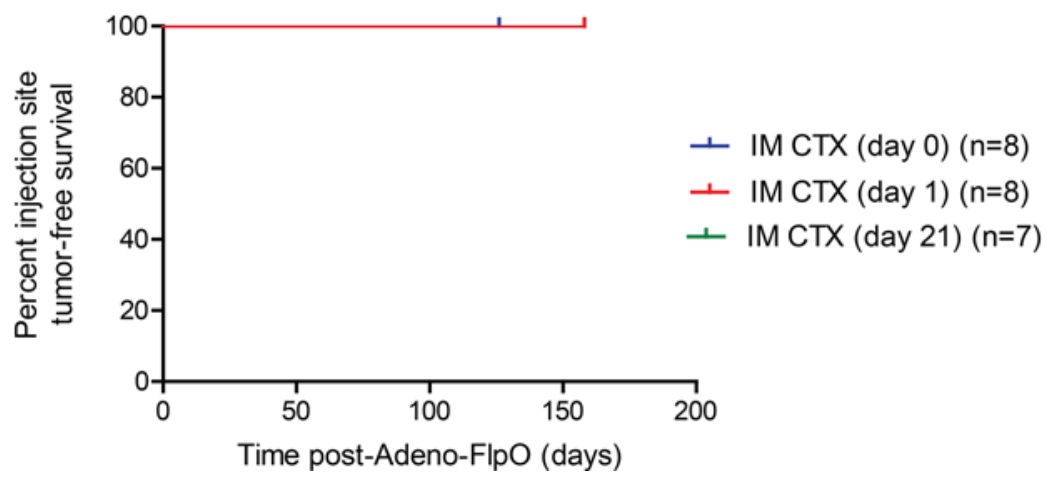

Figure 3. p53 deletion is required for sarcoma formation. (A) Schematic of experiment. R26 $6^{\mathrm{CreER}}$; $\mathrm{p} 53^{\mathrm{fl}}$; $\mathrm{K}^{\mathrm{FRT}}$ mice were treated with adeno-FIpO (deletes Kras) on day 0, followed by IM cardiotoxin on day 0, 1, or 21. (B) Kaplan-Meier survival curve shows that Kras mutation followed by injury does not result in sarcoma formation in the setting of WT p53 in R26 ${ }^{\text {CreER; }}$ p53 ${ }^{\text {fl }}$; $K^{\text {FRT }}$ mice.

by concurrent cardiotoxin 21 days thereafter (Figure 4C). To our surprise, none of the mice developed a sarcoma when injury was delayed (Figure 4D). Similar experiments were conducted where injury was delayed by 1,3 , or 7 days following p53 deletion. There was a trend toward decreasing tumor penetrance and increased time to tumor as the time between p53 deletion and injury increased. For example, only 2 of 11 mice developed a sarcoma when injury was delayed by 3 days, and 0 of 7 mice developed a sarcoma when injury was delayed by 7 days (Figure 4D). Therefore, a single injury to the muscle can promote sarcomagenesis in the setting of systemic p53 deletion only if the injury occurs in close temporal proximity to p53 loss. This result is in sharp contrast to our previous work in Pax $7^{\mathrm{CreER} /+} ; \operatorname{Trp}^{\mathrm{H}} 3^{\mathrm{H} / \mathrm{f}} ; \mathrm{Kras}^{\mathrm{LLL}-\mathrm{G} 12 \mathrm{D} /+}$ (P7KP) mice, where we showed that muscle injury acts as a classic promoter in the setting of concurrent p53 loss and Kras activation by breaking quiescence of tumor-initiating cells (8). Specifically, muscle injury at any time point following genetic recombination of $\mathrm{p} 53$ and $\mathrm{Kras}$ in P7KP mice resulted in acceleration of sarcoma formation (8). The time-dependent aspect of injury on sarcoma formation following p53 deletion alone suggested the possibility of an alternative mechanism.

We next began to investigate differences between $\mathrm{Kras}^{\mathrm{G} 12 \mathrm{D}}$-initiated sarcomas and injury-induced sarcomas in mice with WT Kras. In the context of p53 deletion alone, our hypothesis was that injury might replace Kras mutation by activating the Ras signaling pathway by an alternative means. To test this hypothesis, we treated R26 $6^{\text {CreER; }}$ p53 $3^{\mathrm{fl}} ; \mathrm{K}^{\mathrm{FRT}}$ mice with IP tamoxifen to delete p53 along with adeno-FlpO to activate Kras (Kras-induced) or cardiotoxin (injury-induced) (Figure 5A). We analyzed exome sequencing data from 4 Krasinduced sarcomas and 6 injury-induced sarcomas. We first evaluated non-synonymous mutational frequency, which was not significantly different between the groups (Figure 5B and Supplemental Figure 2A). Moreover, there were no functional mutations found in Ras pathway genes in the injury-induced sarcomas (RAS pathway genes pulled from KEGG database; ref. 10). The mutational frequency in the Kras-induced sarcomas was very similar to sarcomas that arise in Trp53 $3^{\mathrm{A} / \mathrm{fl}} ; \mathrm{Kras}{ }^{\mathrm{LLL}-\mathrm{G} 12 \mathrm{D}}$ mice treated with IM adeno-Cre (11).

We next evaluated CNV frequency between the 2 groups. The frequency of genes with aberrant copy number ratios was almost 2 times higher in injury-induced sarcomas compared with Kras-induced sarcomas $(P=0.019)$ (Figure 5C and Supplemental Figure 2B). Evaluation for recurring amplifications or deletions revealed striking recurrent amplifications in a region of chromosome 6 and/or chromosome 9 (Figure 5D and Supplemental Figure 2C). 
A

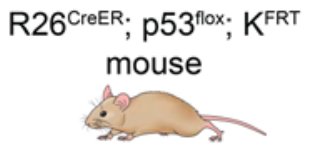

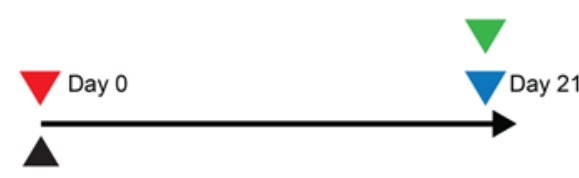

IM Ad-FlpO + CTX

IM Ad-FIpO (activates Kras)

- IP Tamoxifen (deletes p53)

B

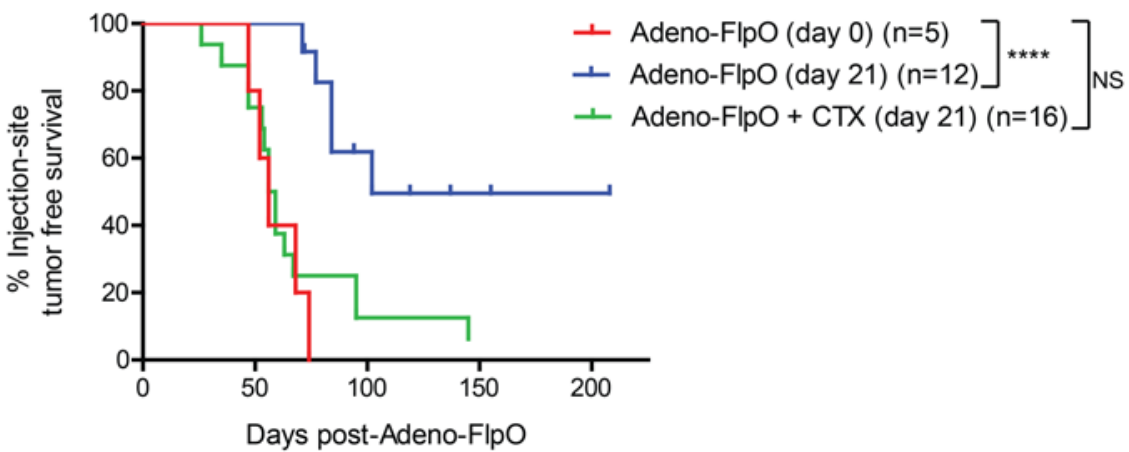

C

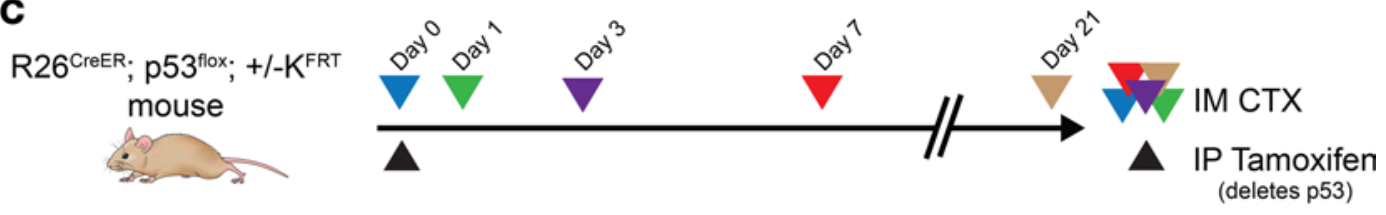

D

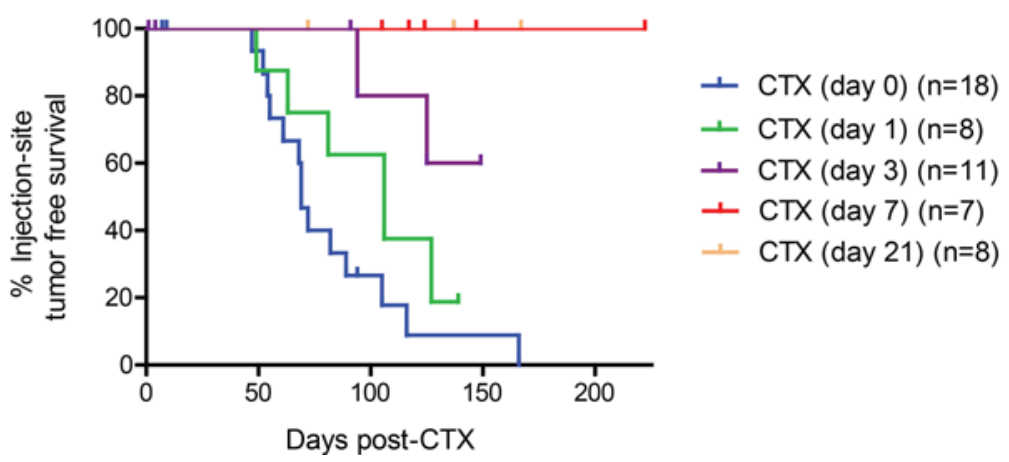

Figure 4. Timing of Kras activation and injury determines the penetrance of sarcoma development in mice following systemic $\mathrm{p} 53$ deletion. (A) Schematic of experiment. R26 $6^{\mathrm{CreER}} ; \mathrm{p} 53^{\mathrm{Fl}} ; \mathrm{K}^{\mathrm{FRT}}$ mice were treated with IP tamoxifen (deletes p53) on day 0 , followed by IM adeno-FlpO on day 0 or 21. An additional group of mice was treated with IM adeno-FIpO and IM cardiotoxin on day 21. (B) Survival curve reveals delayed kinetics and decreased penetrance when Kras signaling is not activated on the same day as p53 deletion. Sarcoma kinetics and penetrance are restored to baseline when IM adeno-FlpO and IM cardiotoxin are coadministered on day $21{ }^{* * * *} P<0.0001$ by log-rank test. (C) Schematic of experiment. R26 ${ }^{\text {CreeR }}$; $53^{\text {fll, }}$ $\mathrm{K}^{\mathrm{FRT}}$ and R26 $6^{\mathrm{CreER}} ; \mathrm{p} 53^{\mathrm{fl}}$ mice were treated with IP tamoxifen on day 0 , followed by IM cardiotoxin on days $0,1,3,7$, and 21. (D) Survival curve shows delayed kinetics and decreased penetrance of sarcoma formation when IM cardiotoxin administration is delayed following IP tamoxifen.

The narrowest amplified region on chromosome 6 includes the Met gene, while the chromosome 9 amplification is most notable for encompassing Yap1 (Figure 6). Both Met and Yap1 have been implicated in mouse models of rhabdomyosarcoma, so we were interested in further exploring this finding $(12,13)$. Primary tumor cell lines were generated from Met- and Yap1-amplified sarcomas, and a Western blot was performed on cell lysates to evaluate protein expression. Met and Yap1 protein expression correlated with the respective gene amplification (Supplemental Figure 3). A quantitative PCR (QPCR) assay was designed to assay Met and Yap1 amplification in a larger number of injury-induced sarcomas from an independent cohort (Supplemental Figure 4, A and B). Injury by either cardiotoxin or application of voltage resulted in significant amplification of Met or Yap1, which was largely mutually exclusive (Figure 7C). Voltage was applied by an similar approach that has been described for in vivo electroporation (11). 
A

$R^{\text {CreeR: }} p 5^{\text {flox. }} K^{\text {FRT }}$ mouse
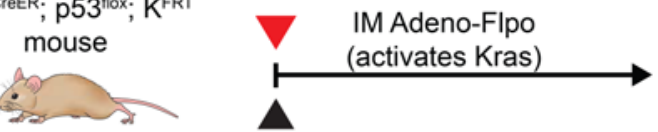

KRAS-INDUCED sarcoma $(n=4)$

R26 CreER; p53 flox; +/-K FRT $^{\text {for }}$ mouse

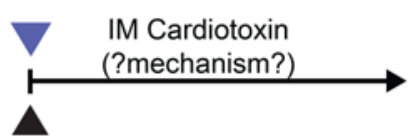

IP Tamoxifen

(deletes p53)

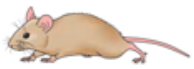

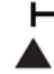

B

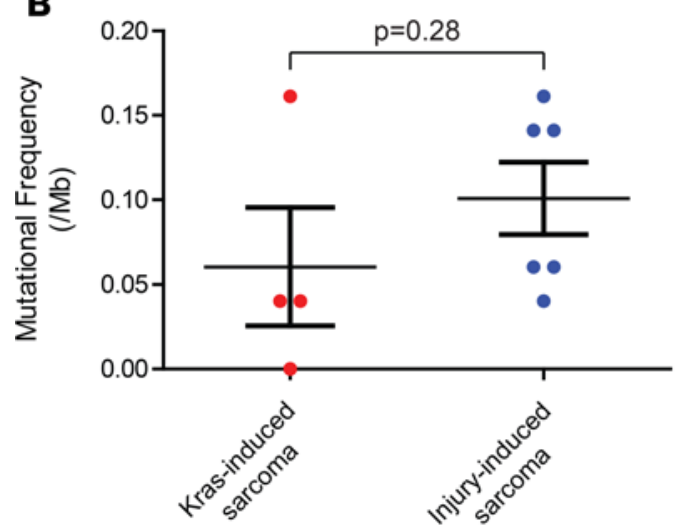

C

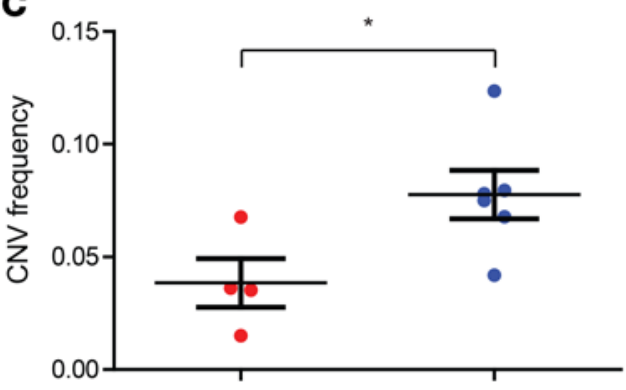

D Mouse

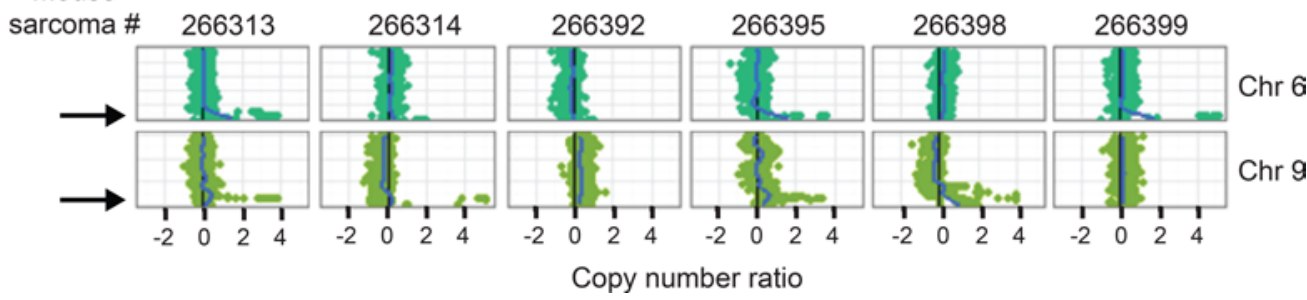

Figure 5. Exome sequencing of Kras- and injury-induced sarcomas. (A) Schematic of experiment. (B) Mutation frequency/Mb plot shows that there is no statistical difference in mutational frequency between the 2 groups. (C) Frequency of copy number variants (CNV) is increased in injury-induced sarcomas versus Kras-induced sarcomas $\left({ }^{*} P=0.019\right)$. (D) Copy number ratio along the length of chromosomes 6 and 9 reveals recurrent regions of focal amplification (arrows).

In our recent experiments in P7KP mice, we showed that Cre-based recombination of p53 and Kras acts as the initiator in muscle satellite cells, and injury serves to break quiescence of the resting stem cells to promote sarcoma formation (8). The finding that injury-mediated sarcomas have characteristic chromosomal amplifications raised the possibility that injury promotes sarcoma development in P7KP mice as a consequence of Met and/or Yap1 amplification. Therefore, we extracted DNA from formalin-fixed, paraffinembedded sarcomas to evaluate for Met and Yap1 amplifications in spontaneous (system tamoxifen treatment alone) and injury-accelerated (systemic tamoxifen + IM cardiotoxin) sarcomas in P7KP mice (Figure 7B). In tumors from P7KP mice, there was no evidence for Met or Yap1 amplification in spontaneous or injury-accelerated sarcomas expressing $\mathrm{Kras}^{\mathrm{G} 12 \mathrm{D}}$ (Figure 7C). Similar to our findings in R26 ${ }^{\mathrm{CreER}}$; $\mathrm{p}^{\mathrm{H}}{ }^{\mathrm{H}}$ mice, Pax $7^{\mathrm{CreER} /+} ; \mathrm{p} 53^{\mathrm{H} / \mathrm{fl}}(\mathrm{P} 7 \mathrm{P})$ mice also developed sarcomas following $\mathrm{p} 53$ deletion with concurrent cardiotoxin injury in the absence of Kras activation (Figure 7A). In P7P mice, Met or Yap1 amplification was also noted in 5 of 6 injury-induced sarcomas (Figure 7C). These data support a differential role for injury in sarcoma formation - as a promoter in the setting of activated Kras and an initiator in the setting of WT Kras.

To further test whether activation of Kras and Met/Yap1 amplification were mutually exclusive, we performed QPCR for Met and Yap1 in Kras ${ }^{\mathrm{G} 12 \mathrm{D}}$-expressing sarcomas from $\mathrm{R} 26^{\mathrm{CreER}} ; \mathrm{p}^{\mathrm{H}}{ }^{\mathrm{H}} ; \mathrm{K}^{\mathrm{FRT}}$ mice generated in previous experiments. There was no amplification of either Met or Yap1 in 4 sarcomas (Supplemental Figure 5A) when Kras was activated 21 days prior to $p 53$ deletion and injury (Figure 2C) and in 8 sarcomas (Supplemental Figure 5B) when p53 was deleted 21 days prior to Kras activation and injury (Figure 4B). 


\begin{tabular}{|c|c|c|c|c|c|c|c|c|c|c|c|}
\hline & & \multicolumn{4}{|c|}{ Kras-induced sarcoma } & \multicolumn{6}{|c|}{ Injury-induced sarcoma } \\
\hline ene & chr & 66300 & 266304 & 266303 & 266306 & 266395 & 266314 & 266313 & 266398 & 266399 & 266392 \\
\hline Met & chr6 & 0.18823 & 0.32946 & 0.06837 & 0.1218 & 3.07404 & 4.89206 & 3.415 & 132 & 0.701722 & 0.8995 \\
\hline Capz & ir6 & $\overline{4479}$ & 0.36229 & 455 & 88169 & 2.64552 & 5.024 & 54669 & 90552 & 1.054 & 269667 \\
\hline $\mathrm{Gm} 1$ & chr6 & -0.0618 & 0.3655 & 995 & & 3.31114 & 4.98475 & & 208 & 1.051 & 0.646 \\
\hline av1 & chr6 & -0.021 & 0.4125 & -0.0466 & -0.2417 & 3.35544 & 4.9532 & 3.174 & 2.9666 & 0 & 0 \\
\hline av2 & chr6 & 0.0734 & 0.34983 & 0.20655 & 0.08629 & 3.28046 & 5.00729 & 3.21944 & 125 & 1.051 & 0.646 \\
\hline Gm4876 & chr6 & 22309 & 0.3 & & & 2.97648 & 5.0329 & 3.4095 & & 0.534 & 7055 \\
\hline Tes & chr6 & 0.22309 & 0.34 & 0.1 & -0 . & 2.97265 & 5.0329 & 3.4095 & 69 & 0.534 & 0.7055 \\
\hline Birc3 & chr9 & 0.14577 & -0.0571 & -0 . & 0.01408 & 3.33033 & 0.20188 & 3.77338 & 0.10 & 5.07247 & -0.08275 \\
\hline$\overline{\mathrm{np}}$ & chr9 & 321 & & & & 3.2166 & 76286 & 3.3705 & 67 & 5.03858 & 7764 \\
\hline Mmp12 & chr9 & -0.0082 & 0.0 & & 0.0 & 3.24633 & 64781 & 3.25717 & 0.3 & 4.962 & -0.12894 \\
\hline Birc2 & chr9 & 0.2219 & 0.0 & & & 3.275 & 0.026519 & 06 & & 3613 & -0.29724 \\
\hline Mmp8 & chr9 & -0.0374 & 0.0 & & -0. & 3.2774 & 0.265591 & 3.21165 & & 5.04265 & -0.13205 \\
\hline Tmem & chr9 & 0.09871 & & & 0.20 & 3.36325 & -0.0983 & 3.6293 & 75 & 4.94443 & -0.33963 \\
\hline \begin{tabular}{|l} 
Dcun1d5 \\
\end{tabular} & chr9 & -0.0203 & -0 . & & -0.0437 & 3.228 & 0.261 & 3.14336 & 417 & 5.0185 & -0.11075 \\
\hline Yap1 & chr9 & 0.10277 & & & 0.11 & 3.5472 & 0.261091 & 3.60542 & 562 & 4.4345 & -0.38763 \\
\hline Mmp7 & chr9 & -0.1049 & 0.02 & & 0.38136 & 3.3929 & -0.00707 & 3.50492 & 0.17 & 4.85155 & -0.31122 \\
\hline Mmp27 & chr9 & 0.11429 & & & & 3.35342 & 0.11255 & 3.15538 & 0.194905 & 5.00936 & -0.2798 \\
\hline Mmp20 & chr9 & -0.1206 & -0.0212 & 0.04224 & -0.1012 & 3.40034 & 0.001217 & 3.42435 & 0.074958 & 4.82083 & -0.18483 \\
\hline & & & & & & & & & -0.04202 & & -0.0012 \\
\hline
\end{tabular}

Figure 6. A list of the top $\mathbf{2 0}$ genes amplified in injury-induced sarcomas. All of the genes were on chromosomes 6 and 9 . Met and Yap1 are highlighted in red. For the QPCR assay, Brca1 was used as a control because it was not amplified or deleted to a significant degree across all samples. Copy number ratio is $\log _{2}$, significant amplification defined as copy number ratio greater than 0.5 (proportional to red saturation), significant deletion less than -0.5 (proportional to blue saturation).

Taken together, these experiments demonstrate that the role of injury in sarcoma development is context dependent. In the setting of p53 deletion and Kras activation, injury functions as a promoter with no evidence of Met or Yap1 amplification. In the absence of Kras activation, injury acts on p53-deleted cells to either generate chromosomal amplifications or to select for rare tumor-initiating cells with Met or Yap1 amplification.

\section{Discussion}

In this study, we utilized dual recombinase technology to explore the complex interplay between the sequential acquisition of genetic mutations and environmental perturbation due to muscle injury in sarcomagenesis. We initiated these experiments to investigate whether injury could rescue the p53-dependent reduction in transformation potential of Kras-activated cells over time (4). We first replicated the results of Young et al. showing that delaying p53 deletion for 21 days after Kras activation results in a dramatic reduction in sarcoma penetrance (Figure 1, B and C). Remarkably, muscle injury at the time of $\mathrm{p} 53$ deletion nearly fully restored the transformation potential of $\mathrm{Kras}^{\mathrm{G} 12 \mathrm{D}}$-expressing cells (Figure 1, B and C), which were confirmed as the cell of origin for these sarcomas by genotyping (Figure 2C). This finding illustrates that the fate of cells harboring activated Kras can be shaped by a change in the microenvironment.

We also made the unexpected observation that systemic p53 deletion alone is sufficient for sarcoma formation in the setting of concomitant injury (Figure 2B and Supplemental Figure 1B). Earlier work had shown that chronic injury promotes sarcoma formation in p53-knockout mice (9), but here we show that a single IM injection of cardiotoxin at the time of systemic p53 deletion is sufficient for sarcomagenesis (Figure 2B and Figure 4D). Interestingly, delaying injury by 21 days after systemic p53 deletion abrogated the ability of injury to promote sarcomagenesis (Figure 4D). Sarcoma formation in the absence of Kras activation suggested that muscle injury might fulfill the criteria of an initiator. We performed exome sequencing to determine whether injury-derived sarcomas harbored mutations in Ras pathway genes. No such mutations were found; however, we observed recurrent amplifications in specific regions of chromosomes 6 and 9 (Figure 5D and Figure 6).

Identical amplicons of chromosomes 6 and 9 were recently described in a mouse model breast cancer with p53 deletion (14), and the chromosome 9 amplicon has been described in mouse models of hepatocellular carcinoma (15), pancreatic cancer (16), and osteosarcoma (17). The chromosome 6 amplicon encompasses Met, a well-characterized oncogene. HGF/Met signaling has been implicated in a mouse 
A

P7P mouse

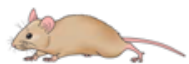

IP Tamoxifen + IM Cardiotoxin

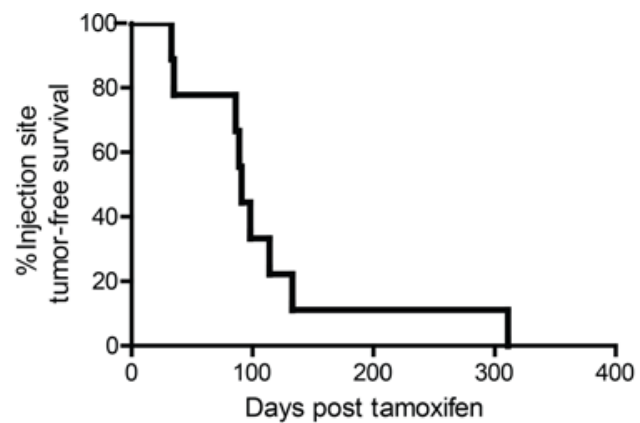

B

P7KP mouse

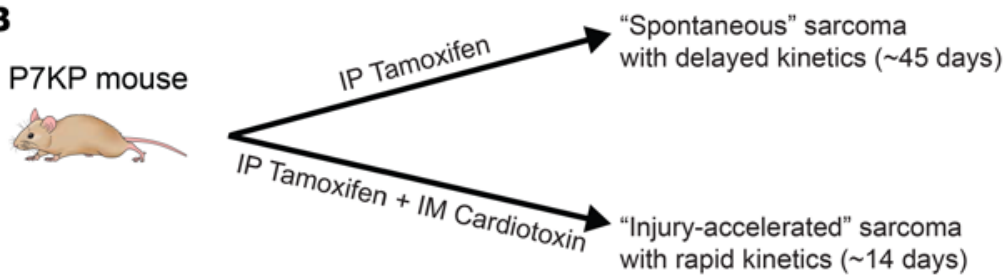

C 80



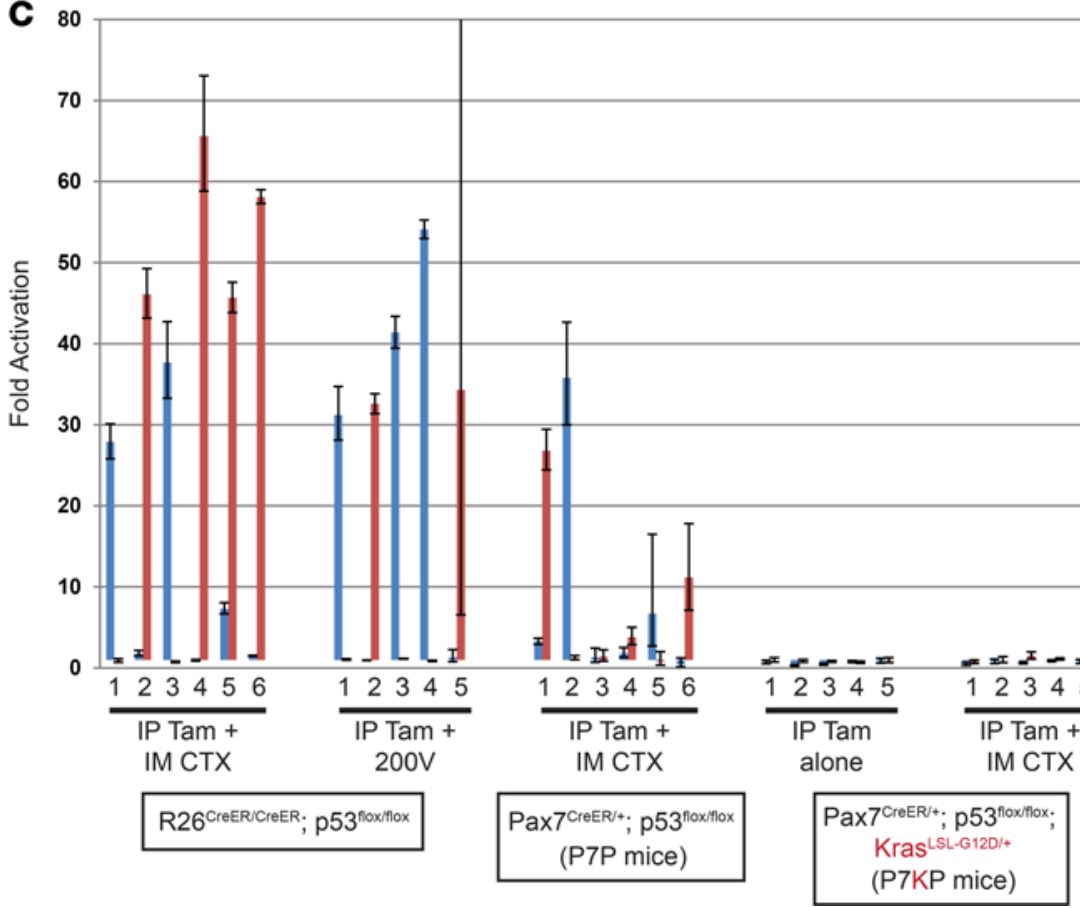

Figure 7. Met and Yap1 amplification is not seen in the setting of activated Kras. (A) Kaplan-Meier curve showing time to tumor formation for Pax $7^{\mathrm{CreER} /+} ; \operatorname{Trp} 53^{\mathrm{fl} / \mathrm{fl}}$ (P7P) mice treated with systemic tamoxifen and concurrent cardiotoxin. All 9 P7P mice developed sarcomas at the injection site. (B) Schematic summarizing results of experiments in Pax $7^{\mathrm{CreER} /+}$; $\operatorname{Trp}^{\mathrm{fl} / \mathrm{fl} \mid}$ K Kras ${ }^{\mathrm{LSL}-\mathrm{C} 12 \mathrm{D} /+}$ (P7KP) mice described in Van Mater et al. (8). (C) A validated QPCR assay was utilized to determine the degree of Met (included in chromosome 6 amplicon) and Yap1 (included in chromosome 9 amplicon) amplification in sarcomas from mice with several different genetic backgrounds. Muscle injury in the absence of Kras mutations was consistently associated with Met and/or Yap1 amplification. QPCR was run in triplicate for each sample, and the fold difference and range are shown by incorporating the standard deviation of the $\Delta \Delta \mathrm{CT}$ value into the fold difference calculation. Tam, tamoxifen; CTX, cardiotoxin; 200V, $200 \mathrm{~V}$ applied via an electric pulse generator to induce muscle injury (11).

model of rhabdomyosarcoma (12), and Met amplifications have been observed in human UPS (18). The Met receptor lies upstream of Ras; thus, HGF/Met signaling represents a viable mechanism to bypass an activating mutation in Kras. The chromosome 9 amplicon includes several intriguing genes, including Yap1, Birc2, Birc3, and a total of 6 MMPs. Among these, Yap1 is particularly interesting, as amplification of Yap1 has been identified as a mechanism to bypass oncogenic Kras addiction in pancreatic cancer cells (16). In a study of murine hepatocellular carcinoma arising from cells with deleted p53, Yap1 amplification was 
noted in tumors with c-myc activation but not in tumors with Ras or Akt mutations (15). This observation provides further support for the notion that Yap1 can function as a Ras bypass pathway in the setting of p53 deletion. A specific role in soft tissue sarcoma is suggested by the finding that concurrent injury with overexpression of a gain-of-function Yap1 mutant in muscle satellite cells leads to rhabdomyosarcoma in mice (13). Additionally, several reports have implicated YAP1 in human soft tissue sarcoma (19-21).

Another key finding of these studies is that the ability of injury to promote sarcoma development depends on the spatial and temporal context of specific gene mutations within tumor-initiating cells. For example, the reduced tumor penetrance in $\mathrm{R} 26^{\mathrm{CreER}}$; p53 $3^{\mathrm{fl}}$; $\mathrm{K}^{\mathrm{FRT}}$ mice after a 21-day delay in Kras activation following systemic p53 deletion could be fully restored when Kras was activated along with muscle injury (Figure 4B). Moreover, in P7KP mice, injury 21 days after systemic activation of oncogenic Kras and p53 deletion promotes sarcomagenesis (8). Therefore, injury promotes sarcoma development in $\mathrm{Kras}^{\mathrm{G} 12 \mathrm{D}}$-expressing cells with $\mathrm{p} 53$ deletion regardless of whether the injury occurs concurrently or 3 weeks after the gene mutations. In contrast, in sarcoma models without Kras ${ }^{\mathrm{G} 12 \mathrm{D}}$ mutation, we made the surprising observation that concurrent, but not delayed, injury leads to sarcoma development with systemic p53 loss (Figure 4D). Because these sarcomas, but not sarcomas initiated from Kras ${ }^{\mathrm{G} 12 \mathrm{D}}$, show amplification of Met or Yap1, the requirement for concurrent injury and p53 loss may reflect differences in Kras versus Met or Yap1 signaling. Remarkably, concurrent injury with cardiotoxin and focal p53 loss by IM injection of adeno-Cre into Trp53 ${ }^{\mathrm{fl} / \mathrm{fl}}$ mice failed to generate sarcomas ( 0 of 12 mice) (Supplemental Figure 6). The requirement for widespread deletion of p53 for concurrent injury to cause sarcoma formation likely reflects the fact that Met and Yap1 amplification is a rare event. Also, it is worth noting that p53 expression is lost in both tumor-initiating cells and in the surrounding microenvironment in R26 CreER; p53 ${ }^{\mathrm{fl}}$ mice. The potential impact of p53 deletion in stromal cells on the phenotype following injury is unknown. The relationship between the timing and extent of p53 deletion and the timing of muscle injury on sarcoma formation warrants further investigation. Additionally, reports of Wnt/ $\beta$ catenin signaling in the setting of muscle injury and crosstalk with Yap1 suggest that a complex interplay of signaling events may mediate the observed phenotype $(22,23)$.

It is interesting to speculate on the clinical relevance of these findings for patients. There are longstanding anecdotal reports of injury playing a role in sarcoma formation (24). These reports led James Ewing to conclude that "sarcoma commonly develops after a single blow" in his 1919 textbook entitled Neoplastic Diseases: A Textbook on Tumors. With the discovery of the genetic basis for cancer, the notion that injury contributes in a meaningful way to sarcomagenesis largely subsided, but studies with Rous sarcoma virus in chickens (25-27) and the phenomenon of feline injection site sarcomas (28) suggest that injury may indeed play a role in tumor formation in certain contexts. Here, we present the most detailed set of experiments to date addressing the role of injury in sarcoma formation in a mammalian system. Our findings suggest that injury must be considered as a potential promoter in genetically engineered cancer models. Furthermore, our results may give clinicians pause when considering a patient's account of injury at a site of sarcoma formation.

In summary, these studies underscore the context-dependent role for injury in sarcoma formation in a mouse model system. In the setting of Kras activation, injury acts a classic promoter to break quiescence of sarcoma-initiating cells. In the setting of WT Kras, muscle injury provides a permissive environment to select for cells with specific chromosome 6 or 9 amplicons. We propose that amplified genes such as Met and Yap1 cooperate with p53 deletion to bypass the requirement for Ras pathway mutations to initiate sarcoma formation. These same gene amplifications may be attractive therapeutic targets in human soft tissue sarcomas.

\section{Methods}

Mouse experiments. Mice were maintained on a mixed 129S4/SvJae and C57BL/6 background. Rosa26 CreER/+ mice and $\mathrm{Kras}^{\text {FRT-STOP-FRT-G12D/+ }}$ mice were obtained from Tyler Jacks (Massachusetts Institute of Technology, Cambridge, Massachusetts, USA), and p53 ${ }^{\mathrm{fl} / \mathrm{fl}}$ mice were obtained from Anton Berns (Netherlands Cancer Institute, Amsterdam, Netherlands). Sarcomas were generated using IP injections of tamoxifen (Sigma-Aldrich) dissolved in $\mathrm{EtOH}$ and diluted in corn oil $(10 \mu \mathrm{l}$ of $20 \mathrm{mg} / \mathrm{ml}$ tamoxifen per gram body weight) (29). Ad5CMVFlpO (adeno-FlpO) was obtained from the University of Iowa Viral Vector Core Facility, and it was prepared and administered as described previously (30). Cardiotoxin (SigmaAldrich) was dissolved in PBS to a concentration of $40 \mu \mathrm{M}$, and a total of $50 \mu 1$ was injected in the gastrocnemius muscle of the mice. Voltage was applied to the muscle of R26 $6^{\mathrm{CreER}} ; \mathrm{p} 53^{\mathrm{H}} ; \mathrm{K}^{\mathrm{FRT}}$ mice as recently described, with the exception that we utilized 200-V pulses, and no DNA was introduced (11). 
Formalin-fixed, paraffin-embedded sarcomas from Pax $7^{\mathrm{CreER} /+}$ mice were generated previously (8) and utilized to assess Met and Yap1 amplification. Mouse genotyping was performed by Transnetyx.

Histology. Tissue samples were fixed in $10 \%$ formalin and embedded in paraffin. Sections were stained with H\&E using a standard protocol. Photomicrographs were obtained on a Leica DM5500B microscope using Leica Application Suite software.

Cell lines. Sarcoma cell lines were generated as described previously (31) and maintained in DMEM (Gibco) with $10 \%$ FBS and 1\% antibiotic-antimycotic (Thermo Fisher Scientific). Cell lines were incubated at $37^{\circ} \mathrm{C}$ with $5 \% \mathrm{CO}_{2}$ in a humidified cell culture incubator.

DNA isolation from cell lines/sarcomas. Freshly isolated sarcomas were placed in RNAlater solution (Invitrogen), and DNA was isolated using the DNeasy Blood and Tissue Kit (QIAGEN). For paraffin-embedded tissue, thin sections were taken by razor blade, and DNA was isolated using the QIAamp DNA FFPE Tissue Kit (QIAGEN).

Assessment of Kras recombination. Kras recombination in sarcoma cell lines was assessed using a PCRbased assay as previously described (11).

Exome sequencing. Preparation and analysis of DNA was performed in an identical fashion as we have recently described (11). In brief, sequencing utilized the Agilent Mouse All Exon kit, followed by high-throughput sequencing on the Illumina HiSeq 2500 platform. Mutation load per Mb was calculated by dividing the count of the filtered somatic variants by the size of the exome target region (49.5 $\mathrm{Mb}$ ). Exome sequencing data have been deposited in the European Nucleotide Archive (PRJEB21980).

Western blot. Cell pellets were resuspended in lysis buffer made of $10 \mathrm{ml}$ RIPA buffer with 1 tablet PhosSTOP (Sigma-Aldrich) and 1 tablet cOmplete Mini Protease Inhibitor Cocktail (Sigma-Aldrich). Lysate was mixed with sample buffer (Laemmli buffer [Bio-Rad] $+5 \% \beta$-mercaptoethanol) and heated to $95^{\circ} \mathrm{C}$ for 5 minutes. Standard SDS-PAGE was performed with $25 \mu$ g protein before transferring to Immobilon-FL PVDF membranes (MilliporeSigma). The membranes were blocked with Odyssey Blocking Buffer in TBS (Li-Cor) and stained using primary antibodies (1:500 rabbit anti-YAP antibody (Cell Signaling Technology, 4912), 1:1,000 rabbit anti-MET antibody (MilliporeSigma, Ab-1003), 1:20,000 mouse anti-actin (BD Biosciences, 612656)) overnight at $4^{\circ} \mathrm{C}$. After washing, the membranes were stained with secondary antibodies (1:15,000 IRDye 800CW goat anti-rabbit IgG (Li-Cor), 1:15,000 IRDye 680RD goat anti-mouse IgG [Li-Cor]) for 1 hour at room temperature. Imaging was performed using the Odyssey CLx Imaging System and ImageStudio software (Li-Cor).

QPCR for Met and Yap1 amplification. Genomic DNA was used as a template in a PCR reaction using PowerUP SYBR Green Master Mix (Applied Biosystems). Met, Yap1, and Brca1 primers were generated using Primer 3 software, and the QuantStudio 6 Flex Real-Time PCR System (Applied Biosystems) was used for cycling and data analysis. Primers were as follows: Met DNA, forward: AATATCCTCCAAGCCGCGTA; Met DNA, reverse: TGATGGGGAATGGACAGACT; Yap1 DNA, forward: CAAATGTGGACCTTGGCACA; Yap1 DNA, reverse: CCCTCACAGACTCAGAGTGG; Brca1 DNA, forward: CGGATGCCAAGAAGAACGAG; Brca1 DNA, reverse: GTTCCTGTTCTCTGAGGGCT.

Statistics. For tumor kinetics studies, Kaplan-Meier analysis was performed, and the log-rank test was used to determine statistical significance. Mann-Whitney $U$ test was performed to compare the means of 2 groups. Significance was assumed for $P<0.05$. All calculations were performed using Prism 6 (GraphPad). For the quantitative PCR studies expressing fold change in Met and Yap1, all samples were run in triplicate, and the confidence interval is shown using methods described in the Applied Biosystems user manual.

Study approval. All animal experiments were performed according to a protocol approved by the Duke University Institutional Animal Care and Use Committee (A109-13-04).

\section{Author contributions}

DVM and DGK were responsible for overall research design, data analysis, and writing the manuscript. DVM, EX, LA, MS, WH, NW, YM, CL, and LH were responsible for conducting experiments. AR and $\mathrm{SD}$ were responsible for the sequencing analysis.

\section{Acknowledgments}

We thank Tyler Jacks for providing the Rosa26 $6^{\mathrm{CreER} /+}$ and $\mathrm{Kras}^{\mathrm{Frt}-S T O P-F r t-G 12 \mathrm{D} /+}$ mice. We thank Anton Berns for providing the $\mathrm{p} 53^{\mathrm{f} / \mathrm{fl}}$ mice. Additional sequencing support was provided by Kouros Owzar, Dadong Zhang, and Xiaodi Qin of the Duke Cancer Institute Bioinformatics Shared Resource. This work was 
supported by 1R35CA197616 from the National Cancer Institute to DGK and a Sarcoma Alliance for Research through Collaboration (SARC) Career Development Award to DVM. We thank members of the Kirsch laboratory for advice and suggestions.

Address correspondence to: David Kirsch, Duke University Medical Center, DUMC Box 91006, Durham North Carolina 27710, USA. Phone: 919.681.8586; Email: david.kirsch@duke.edu.

1. Borden EC, et al. Soft tissue sarcomas of adults: state of the translational science. Clin Cancer Res. 2003;9(6):1941-1956.

2. Kirsch DG, et al. A spatially and temporally restricted mouse model of soft tissue sarcoma. Nat Med. 2007;13(8):992-997.

3. Mito JK, et al. Cross species genomic analysis identifies a mouse model as undifferentiated pleomorphic sarcoma/malignant fibrous histiocytoma. PLoS ONE. 2009;4(11):e8075.

4. Young NP, Crowley D, Jacks T. Uncoupling cancer mutations reveals critical timing of p53 loss in sarcomagenesis. Cancer Res. 2011;71(11):4040-4047.

5. Moding EJ, et al. Atm deletion with dual recombinase technology preferentially radiosensitizes tumor endothelium. J Clin Invest. 2014;124(8):3325-3338.

6. Moding EJ, et al. Tumor cells, but not endothelial cells, mediate eradication of primary sarcomas by stereotactic body radiation therapy. Sci Transl Med. 2015;7(278):278ra34.

7. Raymond CS, Soriano P. High-efficiency FLP and PhiC31 site-specific recombination in mammalian cells. PLoS ONE. 2007;2(1):e162.

8. Van Mater D, et al. Acute tissue injury activates satellite cells and promotes sarcoma formation via the HGF/c-MET signaling pathway. Cancer Res. 2015;75(3):605-614.

9. Camboni M, Hammond S, Martin LT, Martin PT. Induction of a regenerative microenvironment in skeletal muscle is sufficient to induce embryonal rhabdomyosarcoma in p53-deficient mice. J Pathol. 2012;226(1):40-49.

10. Kanehisa M, Goto S. KEGG: Kyoto encyclopedia of genes and genomes. Nucleic Acids Res. 2000;28(1):27-30

11. Huang J, et al. Generation and comparison of CRISPR-Cas9 and Cre-mediated genetically engineered mouse models of sarcoma. Nat Commun. 2017;8:15999.

12. Sharp R, et al. Synergism between INK4a/ARF inactivation and aberrant HGF/SF signaling in rhabdomyosarcomagenesis. Nat Med. 2002;8(11):1276-1280.

13. Tremblay AM, et al. The Hippo transducer YAP1 transforms activated satellite cells and is a potent effector of embryonal rhabdomyosarcoma formation. Cancer Cell. 2014;26(2):273-287.

14. Tao L, Xiang D, Xie Y, Bronson RT, Li Z. Induced p53 loss in mouse luminal cells causes clonal expansion and development of mammary tumours. Nat Commun. 2017;8:14431.

15. Zender L, et al. Identification and validation of oncogenes in liver cancer using an integrative oncogenomic approach. Cell. 2006;125(7):1253-1267.

16. Kapoor A, et al. Yap1 activation enables bypass of oncogenic Kras addiction in pancreatic cancer. Cell. 2014;158(1):185-197.

17. Ma O, et al. MMP13, Birc2 (cIAP1), and Birc3 (cIAP2), amplified on chromosome 9, collaborate with p53 deficiency in mouse osteosarcoma progression. Cancer Res. 2009;69(6):2559-2567.

18. Schmitz K, et al. MET gene copy number alterations and expression of MET and hepatocyte growth factor are potential biomarkers in angiosarcomas and undifferentiated pleomorphic sarcomas. PLoS ONE. 2015;10(4):e0120079.

19. Fullenkamp CA, et al. TAZ and YAP are frequently activated oncoproteins in sarcomas. Oncotarget. 2016;7(21):30094-30108.

20. Eisinger-Mathason TS, et al. Deregulation of the Hippo pathway in soft-tissue sarcoma promotes FOXM1 expression and tumorigenesis. Proc Natl Acad Sci USA. 2015;112(26):E3402-E3411.

21. Cancer Genome Atlas Research Network. Electronic address: elizabeth.demicco@sinaihealthsystem.ca, Cancer Genome Atlas Research Network. Comprehensive and Integrated Genomic Characterization of Adult Soft Tissue Sarcomas. Cell. 2017;171(4):950-965.e28.

22. Huraskin $\mathrm{D}$, et al. Wnt/ $\beta$-catenin signaling via Axin2 is required for myogenesis and, together with YAP/Taz and Tead1, active in IIa/IIx muscle fibers. Development. 2016;143(17):3128-3142.

23. Rudolf A, et al. $\beta$-Catenin activation in muscle progenitor cells regulates tissue repair. Cell Rep. 2016;15(6):1277-1290.

24. Coley WB. II. The influence of injury upon the development of sarcoma. Ann Surg. 1898;27(3):259-284.

25. Dolberg DS, Hollingsworth R, Hertle M, Bissell MJ. Wounding and its role in RSV-mediated tumor formation. Science. 1985;230(4726):676-678.

26. Sieweke MH, Thompson NL, Sporn MB, Bissell MJ. Mediation of wound-related Rous sarcoma virus tumorigenesis by TGFbeta. Science. 1990;248(4963):1656-1660.

27. Martins-Green M, Boudreau N, Bissell MJ. Inflammation is responsible for the development of wound-induced tumors in chickens infected with Rous sarcoma virus. Cancer Res. 1994;54(16):4334-4341.

28. Porcellato I, et al. Feline injection-site sarcoma. Vet Pathol. 2017;54(2):204-211.

29. Dodd RD, Añó L, Blum JM, Li Z, Van Mater D, Kirsch DG. Methods to generate genetically engineered mouse models of soft tissue sarcoma. Methods Mol Biol. 2015;1267:283-295.

30. Lee CL, et al. Generation of primary tumors with Flp recombinase in FRT-flanked p53 mice. Dis Model Mech. 2012;5(3):397-402.

31. Sachdeva M, et al. MicroRNA-182 drives metastasis of primary sarcomas by targeting multiple genes. J Clin Invest. 2014;124(10):4305-4319. 